\title{
Graph Concatenations to Derive Weighted Fractal Networks
}

\author{
Zhanqi Zhang (D) and Yingqing Xiao \\ College of Mathematics and Econometrics, Hunan University, Changsha 410082, China
}

Correspondence should be addressed to Zhanqi Zhang; mdyd1991@hnu.edu.cn

Received 29 December 2019; Revised 13 June 2020; Accepted 23 June 2020; Published 18 July 2020

Academic Editor: Ning Cai

Copyright ( $) 2020$ Zhanqi Zhang and Yingqing Xiao. This is an open access article distributed under the Creative Commons Attribution License, which permits unrestricted use, distribution, and reproduction in any medium, provided the original work is properly cited.

\begin{abstract}
Given an initial weighted graph $G_{0}$, an integer $m>1$, and $m$ scaling factors $f_{1}, \ldots, f_{m} \in(0,1)$, we define a sequence of weighted graphs $\left\{G_{k}\right\}_{k=0}^{\infty}$ iteratively. Provided that $G_{k-1}$ is given for $k \geq 1$, we let $G_{k-1}^{(1)}, \ldots, G_{k-1}^{(m)}$ be $m$ copies of $G_{k-1}$, whose weighted edges have been scaled by $f_{1}, \ldots, f_{m}$, respectively. Then, $G_{k}$ is constructed by concatenating $G_{0}$ with all the $m$ copies. The proposed framework shares several properties with fractal sets, and the similarity dimension $d_{\text {fract }}$ has a great impact on the topology of the graphs $G_{k}$ (e.g., node strength distribution). Moreover, the average geodesic distance of $G_{k}$ increases logarithmically with the system size; thus, this framework also generates the small-world property.
\end{abstract}

\section{Introduction}

For the past two decades, various networked systems and their models have been studied intensively by researchers from many different scientific fields. Examples include neural networks, protein networks, metabolic networks, phone call networks, the Internet and the World Wide Web, social networks of acquaintance, and many others. The graph models of networked systems such as these often consist of millions or even billions of nodes and have large-scale statistical properties. Due to the abundance of systems of this sort and the increased computing powers, complex networks are currently very hot and attractive research objects, and they define a powerful framework for describing and analyzing the real-world networks. In comparison with regular networks and random networks, the structure of complex networks is irregular, complex, and usually dynamically evolving in time. Complex networks possess all or part of the remarkable properties including small-world effect, scale-free property, correlations in the node degrees, and community structure, which reproduce topological properties of most of the realworld networks considered. One can refer to [1-5] for a detailed exposition of the theory of complex networks.

In the study of complex networks, one of the topics is to construct models mimicking real-world networks. Some construction algorithms are based on the fixed fractal sets or merely the ideas taken from the fractal construction. In [6], Barabási et al. presented a model that constructs a sequence of networks in a deterministic fashion and proved the model generates the scale-free property. In [7], Zhang et al. constructed a class of networks named incompatibility networks based on the structure of the Sierpiński gasket and showed that the networks are scale-free and small-world. In [8], the authors proposed a method converting the Koch curve into a family of deterministic networks called Koch networks, which also possess general properties observed in real-world networks. Inspired by the works of mapping fractal sets into complex networks and in order to generalize the former constructions, Carletti and Righi defined a class of weighted complex networks via an explicit algorithm in [9]. Their framework shares several properties with fractal sets, and the procedure of constructing the networks is similar to constructing a sequence of sets by the iterated function system [10]. They hereby called this framework weighted fractal networks, WFNs for short. Recently, in [11], Li et al. introduced a method of generating a family of growing symmetrical tree networks $\left\{T_{k}\right\}_{k=0}^{\infty}$ from a symmetrical tree $T_{0}$, where $T_{k}$ is constructed by replacing each edge of $T_{k-1}$ with a reduced scale of $T_{0}$. They studied the asymptotic behavior of the average geodesic distance of $T_{k}$. In [12], Xi and Ye did a similar work, whereas the initial graph turns into a directed tree. 
In this paper, we introduce a method which generates a class of weighted graphs based on a weighted graph and some parameters. Our framework is different from the construction in [9]; it, however, also shares some properties with fractal sets, hereby named weighted fractal networks. As for the model introduced in [9], the topological properties of the networks are mainly determined by the scaling factor, the number of copies, and the order and size of the initial network, whereas other topologies of the initial network have only a small impact on the properties of their framework. In our case, the initial graph plays a dominant role in the construction and topologies of the weighted graphs. In Section 2, we will introduce the model and show some applications of it. In Section 3, we study several topological properties of the graphs, including the average weighted geodesic distance and the node strength distribution. We found that the average geodesic distance increases logarithmically with the system size; thus, the weighted fractal networks generate the small-world property. Besides, the similarity dimension plays a relevant role in the topologies of the weighted fractal networks.

\section{Weighted Fractal Networks}

2.1. The Model. Let us fix a positive integer $m>1$ and $m$ positive real numbers $f_{1}, \ldots, f_{m} \in(0,1)$. Let $G_{0}=\left(V\left(G_{0}\right), E\left(G_{0}\right)\right)$ be an initial weighted graph satisfying three conditions: $G_{0}$ has no loops or parallel edges; $G_{0}$ is connected; and the cardinality of nodes in $G_{0}$, denoted by $n$, satisfies $n=\#\left(V\left(G_{0}\right)\right)>m$. One of the nodes has been labeled as the beginning node and is denoted by $s_{0} . m$ other nodes have been labeled as end nodes and are denoted by $v_{1}, v_{2}, \ldots, v_{m}$, respectively.

We construct a sequence of weighted graphs $\left\{G_{k}\right\}_{k=0}^{\infty}$ iteratively. $G_{0}$ is given. We let $G_{0}^{(1)}, \ldots, G_{0}^{(m)}$ be $m$ copies of $G_{0}$. For each $i \in\{1, \ldots, m\}$, weighted edges of $G_{0}^{(i)}$ have been scaled by $f_{i}$, and the node in the $G_{0}^{(i)}$ image of the beginning node $s_{0}$ is denoted by $s_{0}^{(i)}$. Concatenate $G_{0}$ with $G_{0}^{(i)}$ by merging node $v_{i}$ and node $s_{0}^{(i)}$ into a single new node, denoted by $v_{i}^{(1)}$, for $i=1, \ldots, m$. Then, we obtain the new graph $G_{1}$, of which $G_{0}$ is a subgraph. $s_{0}$ in the new graph is also labeled as a beginning node, and we denote it by $s_{1}$ when talking about $G_{1}$.

Suppose $G_{k-1}$ is given, we define $G_{k}$ similarly. Let $G_{k-1}^{(1)}, \ldots, G_{k-1}^{(m)}$ be $m$ copies of $G_{k-1}$. For each $i \in\{1, \ldots, m\}$, weighted edges of $G_{k-1}^{(i)}$ have been scaled by $f_{i}$, and the node in the $G_{k-1}^{(i)}$ image of the beginning node $s_{k-1}$ is denoted by $s_{k-1}^{(i)}$. Concatenate $G_{0}$ with $G_{k-1}^{(i)}$ by merging node $v_{i}$ and node $s_{k-1}^{(i \overline{)})}$ into a single new node, denoted by $v_{i}^{(k)}$, for $i=1, \ldots, m$. We obtain graph $G_{k} . s_{0}$ in the new graph $G_{k}$ is labeled as a beginning node and is also denoted by $s_{k}$. Two examples are stated for illustration (see Figures 1 and 2).

The similarity dimension $d_{\text {fract }}$ of the WFN is the real number satisfying

$$
\sum_{i=1}^{m}\left(f_{i}\right)^{d_{\text {fract }}}=1 .
$$

If $f_{1}=\cdots=f_{m}=f \in(0,1)$, then $d_{\text {fract }}=-\log m / \log f$.
Our framework supplies a general way of constructing complex networks with respect to plenty of fractal sets, e.g., Sierpinski gasket, uniform Cantor set [13], generalized Koch curves [14], and Cantor dust [15]. Roughly speaking, using this framework, we could construct complex networks with remarkable properties (e.g., small-world effect and scale-free property) from a lot of self-similar sets, for which the iterated function systems satisfy the open set condition. The approach provides an alternative way to study fractals via the theory of complex networks, and vice versa.

2.2. Generalized Koch Curve. Let us fix a positive integer $p \in \mathbb{N}$. We define an operation as follows: partition each existing line segment into $2 p+1$ segments, which are consecutively numbered $1, \ldots, 2 p+1$ from one endpoint to the other; replace each even-numbered segment by the other two sides of the equilateral triangle based on the removed segment. Let $H_{p, 0}$ be a line segment of unitary length. For $t>0$, we obtain $H_{p, t}$ by performing the operation on $H_{p, t-1}$. The sequence of curves $H_{p, t}$ approaches a limit curve, called the generalized Koch curve. With respect to this fractal set, we set $m=3 p+1$ and $f_{1}=\cdots=f_{3 p+1}=1 /(2 p+1)$. Obviously, the similarity dimension $d_{\text {fract }}=\log (3 p+$ $1) / \log (2 p+1)$ is equal to the Hausdorff dimension of the generalized Koch curve. The initial weighted graph $G_{0}$ is as follows: $G_{0}$ consists of $3 p+2$ nodes, one of which has been labeled the beginning node, and the others have been labeled end nodes. Each end node and the beginning node are adjacent with an edge of unitary weight. There is a one-toone mapping from the segments of $H_{p, 1}$ to the end nodes of $G_{0}$. Two end nodes are adjacent if and only if their inverse images in $H_{p, 1}$ are connected. The edges that join two end nodes have a weight $1 /(2 p+1)$. Figure 3 shows the structure of $H_{2,2}$ and the corresponding initial graph $G_{0}$.

2.3. Cantor Dust. Define an operation as follows: divide each existing square into 16 identical squares, which are consecutively numbered $1, \ldots, 16$ from left to right and from top to bottom. Set $A=\{2,3,5,8,9,12,14,15\}$. Remove the squares whose numbers belong to $A$, and we obtain 5 squares. Let $S_{0}$ be a unit square. For $t>0$, we obtain $S_{t}$ by performing the operation on $S_{t-1}$. The limit set of the sequence $\left\{S_{t}\right\}_{t=0}^{\infty}$ is Cantor dust. With respect to this fractal set, we set $m=5, f_{1}=\cdots=f_{4}=1 / 4$, and $f_{5}=1 / 2$. Once again, the similarity dimension and the Hausdorff dimension of the Cantor dust coincide. The initial weighted graph $G_{0}$ is as follows: $G_{0}$ consists of 14 nodes, one of which has been labeled the beginning node, and 5 others have been labeled end nodes. Assume $V\left(G_{0}\right)=\left\{s_{0}, v_{1}, \ldots, v_{5}, u_{1}, \ldots, u_{8}\right\}$. The beginning node $s_{0}$ and other nodes are adjacent with an edge of unitary weight. For each node $x \in V\left(G_{0}\right) \backslash\left\{s_{0}, v_{5}\right\}, x$ is connected to $v_{5}$ with an edge of weight $1 / 4+1 / 8$. There is a one-to-one mapping $\psi$ from $V\left(G_{0}\right) \backslash\left\{s_{0}, v_{5}\right\}$ to the squares in $S_{1}$ whose numbers belong to $A \cup\{1,4,13,16\}$ satisfying $\psi\left(\left\{u_{1}, \ldots, u_{8}\right\}\right)=A$. The nodes in $V\left(G_{0}\right) \backslash\left\{s_{0}, v_{5}\right\}$ are connected to one another with edges of weight $1 / 8+1 / 8$ if their images in $S_{1}$ are connected (see Figure 4 for illustration). 


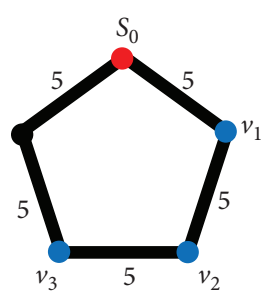

(a)

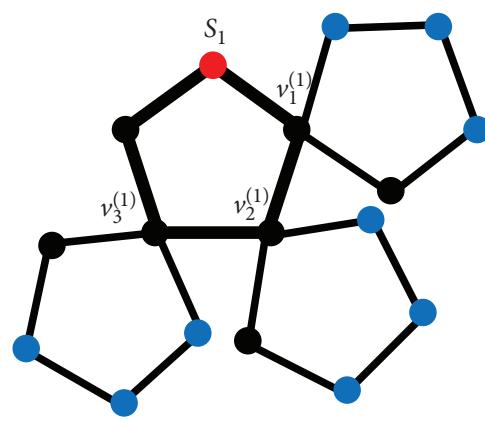

(b)

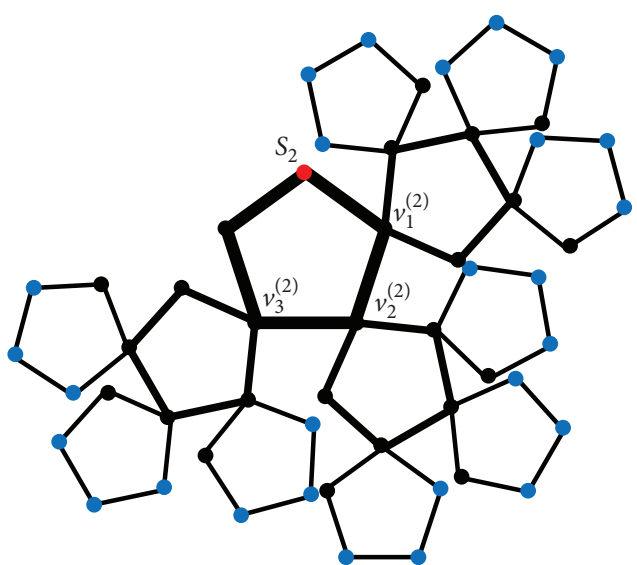

(c)

FIGURE 1: From the left to the right, $G_{0}, G_{1}$, and $G_{2}$. $G_{0}$ is a 5 -cycle, whose edges have weight 5 . For each $i \in\{0,1,2\}$, the red point of $G_{i}$ denotes the beginning node, and the blue points of $G_{i}$ denote the end nodes. Set $f_{1}=f_{2}=f_{3}=0.6$. Thickness of the lines reproduces edge weights: the thicker the line, the larger the weight.
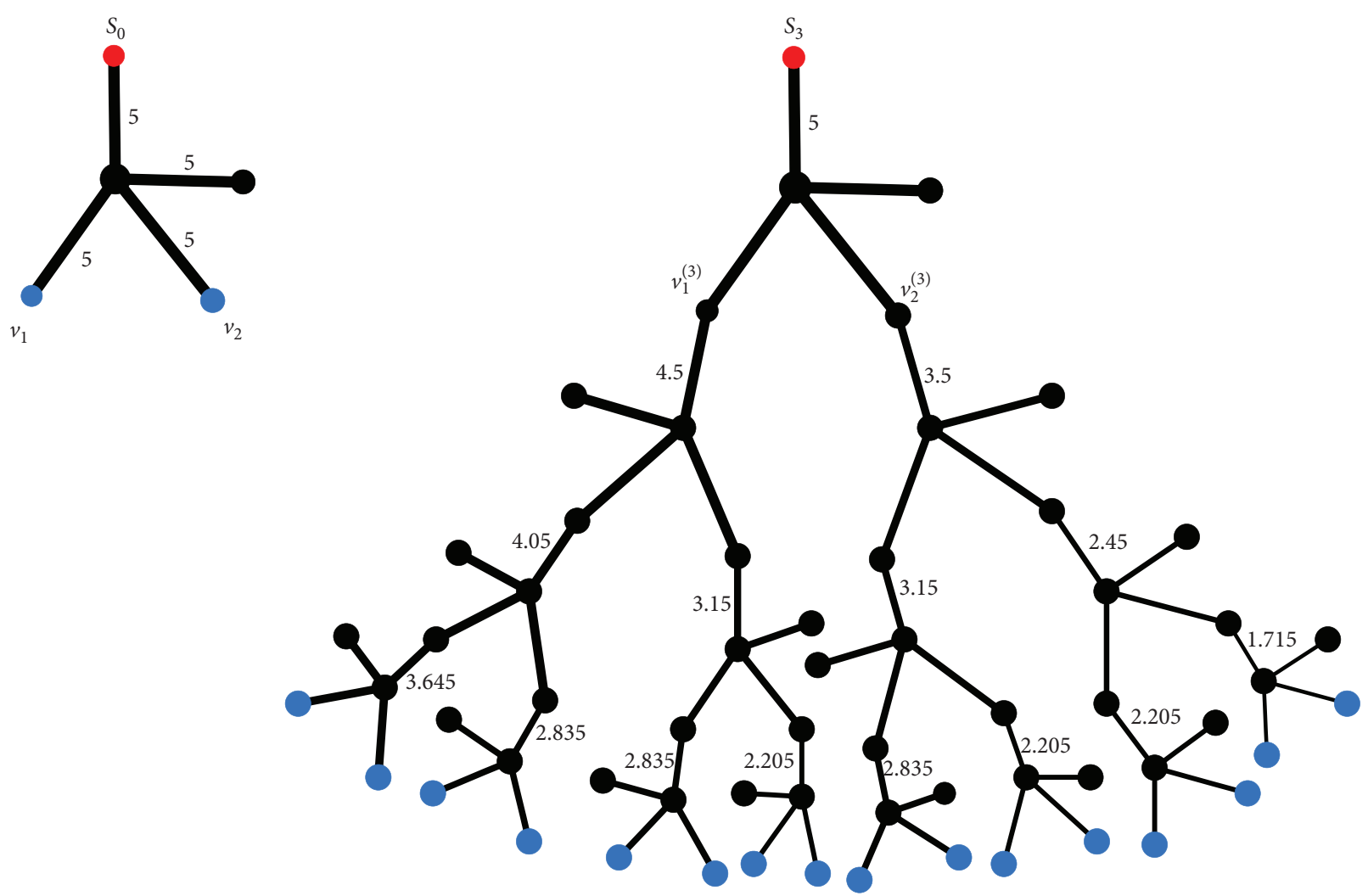

(a)

(b)

FIgURe 2: The graphical representations of (a) $G_{0}$ and (b) $G_{3} . G_{0}$ has 2 end nodes and 4 edges of weight 5 . We set $f_{1}=0.9$ and $f_{2}=0.7$. Thickness of the lines reproduces edge weights: the thicker the line, the larger the weight.

Due to the self-similarity of the weighted fractal networks and the scaling factors $f_{1}, \ldots, f_{m} \in(0,1)$, our approach may have some applications in the spread of some infections, connection of the social communication, airflows in mammalian lungs, phone call networks, and so on. The framework also provides an alternative to explain the frequent appearance of fractal phenomena in nature and society. 

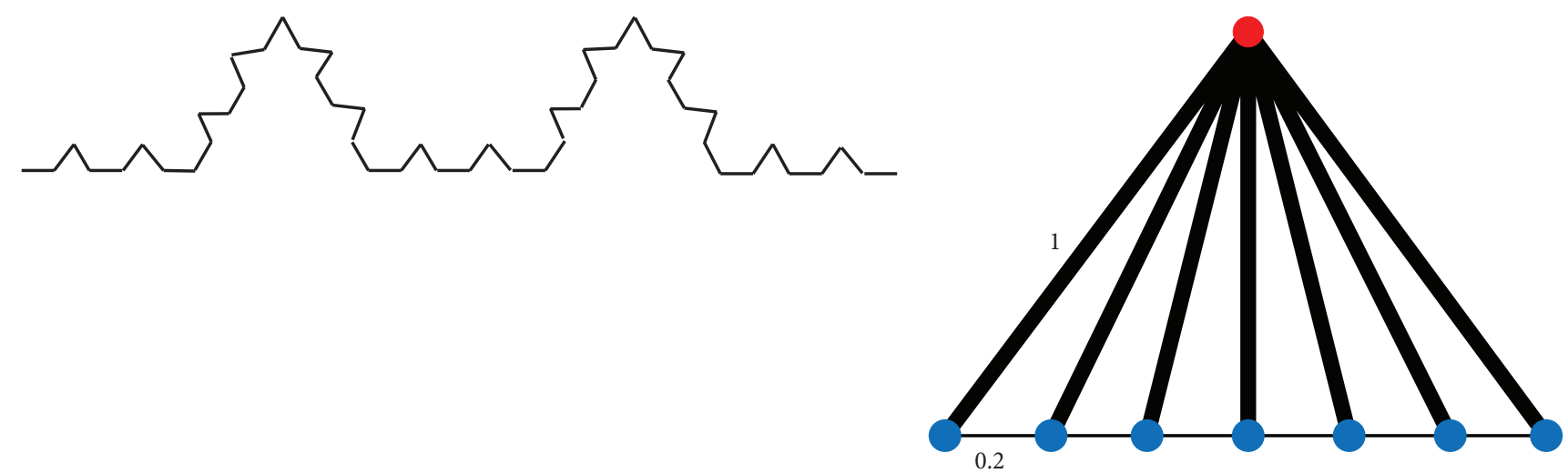

(a)

(b)

FIgURe 3: (a) The first two generations of the construction for the generalized Koch curve with $p=2$. (b) The initial weighted graph $G_{0}$.

\begin{tabular}{|c|c|c|c|}
\hline 1 & 2 & 3 & 4 \\
\hline 5 & 6 & 7 & 8 \\
\hline 9 & 10 & 11 & 12 \\
\hline 13 & 14 & 15 & 16 \\
\hline
\end{tabular}

(a)

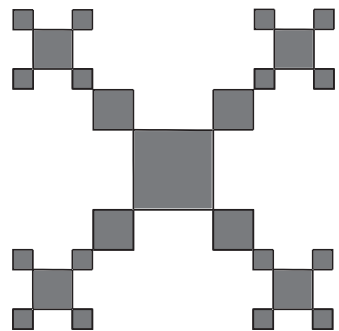

(b)

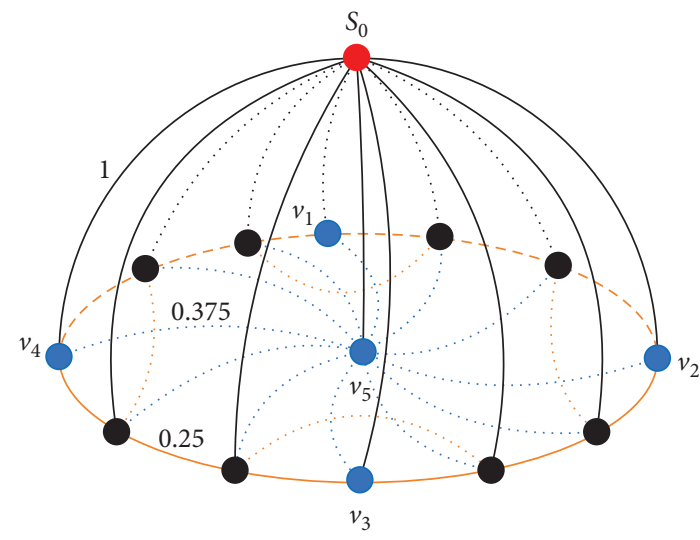

(c)

Figure 4: In $G_{0}$, black lines denote edges of weight 1 , blue lines denote edges of weight 0.375 , and orange lines denote edges of weight 0.25 . (a) $S_{1}$. (b) $S_{2}$. (c) $G_{0}$.

\section{Topological Properties}

In this section, we characterize the topology of the weighted graphs $G_{k}$ for all $k \geq 0$. Let $N_{k}$ be the cardinality of nodes in $G_{k}$ and $E_{k}$ be the cardinality of edges in $G_{k}$. One can easily verify that

$$
\begin{aligned}
& N_{k}=\frac{m^{k+1}-1}{m-1} n-\frac{m^{k}-1}{m-1} m, \\
& E_{k}=\frac{m^{k+1}-1}{m-1} E_{0} .
\end{aligned}
$$

The degree of node $v$ in graph $G$ is the cardinality of edges of $G$ incident with $v$. The sum of degrees of all nodes in $G$ equals to $2 \#(E(G))$ ([16], Theorem 1.1). Note that, for any graph $G$, we denote the class of all nodes in $G$ by $V(G)$ and denote by $E(G)$ the class of all edges in $G$. The cardinal numbers of $V(G)$ and $E(G)$ are called the order and size of $G$, respectively. Thus, the asymptotic behavior of the average degree of $G_{k}$ satisfies

$$
\frac{2 E_{k}}{N_{k}}=\frac{2\left(m^{k+1}-1\right) E_{0}}{m^{k+1}(n-1)-(n-m)} \underset{k}{\longrightarrow \infty} \frac{2 E_{0}}{n-1} .
$$

3.1. Average Weighted Geodesic Distance. Let $d^{(k)}(x, y)$ denote the weighted geodesic distance between nodes $x$ and $y$ in $G_{k}$. Set $d(\cdot, \cdot)=d^{(0)}(\cdot, \cdot)$ for convention. Define

$$
\Lambda_{k}=\sum_{x, y \in G_{k}} d^{(k)}(x, y), \quad \text { for } k \geq 0 .
$$

Thus, the average weighted geodesic distance of $G_{k}$ is given by

$$
\delta_{k}=\frac{\Lambda_{k}}{N_{k}\left(N_{k}-1\right)} .
$$

For $k \geq 0$, we define

$$
\lambda_{k}=\sum_{x \in G_{k}} d^{(k)}\left(x, s_{k}\right),
$$


i.e., the sum of all weighted geodesic distances of the nodes in $G_{k}$ from the beginning node $s_{k}$. Define $F=\sum_{i=1}^{m} f_{i}$. Set

$$
\begin{aligned}
& \lambda_{0}^{\prime}=\sum_{i=1}^{m} d\left(v_{i}, s_{0}\right), \\
& \lambda_{0}^{\prime \prime}=\lambda_{0}-\lambda_{0}^{\prime} .
\end{aligned}
$$

Lemma 1. For $k \geq 1$, we have

$$
\lambda_{k}=F^{k} \lambda_{0}+\left(\sum_{t=0}^{k-1} F^{k-1-t} N_{t}\right) \lambda_{0}^{\prime}+\left(\sum_{t=0}^{k-1} F^{t}\right) \lambda_{0}^{\prime \prime} .
$$

The asymptotic behavior of $\lambda_{k}$ satisfies

$$
\lim _{k \rightarrow \infty} \frac{\lambda_{k}}{m^{k}}=\frac{m(n-1)}{(m-1)(m-F)} \lambda_{0}^{\prime} .
$$

Proof. By the construction of $G_{k}$, we obtain that

$$
\begin{aligned}
\lambda_{k} & =\sum_{i=1}^{m}\left(f_{i} \lambda_{k-1}+N_{k-1} d\left(v_{i}, s_{0}\right)\right)+\lambda_{0}^{\prime \prime} \\
& =F \lambda_{k-1}+N_{k-1} \lambda_{0}^{\prime}+\lambda_{0}^{\prime \prime} .
\end{aligned}
$$

By direct calculation, we obtain equation (8). Since $F<m$, we have

$$
\lim _{k \rightarrow \infty} \frac{F^{k} \lambda_{0}+\left(\sum_{t=0}^{k-1} F^{t}\right) \lambda_{0}^{\prime \prime}}{m^{k}}=0 .
$$

Thus, in order to prove equation (9), it suffices to prove

$$
\lim _{k \rightarrow \infty} \frac{\sum_{t=0}^{k-1} F^{k-1-t} N_{t}}{m^{k}}=\frac{m(n-1)}{(m-1)(m-F)} .
$$

However,

$$
\begin{aligned}
\lim _{k \rightarrow \infty} \frac{\sum_{t=0}^{k-1} F^{k-1-t} N_{t}}{m^{k}} & =\frac{1}{F} \lim _{k \rightarrow \infty} \frac{\sum_{t=0}^{k-1} N_{t} / F^{t}}{(m / F)^{k}} \\
& =\frac{1}{F} \lim _{k \rightarrow \infty} \frac{N_{k-1} / F^{k-1}}{(m / F)^{k}-(m / F)^{k-1}} \\
& =\frac{1}{m-F} \lim _{k \rightarrow \infty} \frac{N_{k-1}}{m^{k-1}} \\
& =\frac{m(n-1)}{(m-1)(m-F)} .
\end{aligned}
$$

The second equality uses O. Stolz's formula. The proof is complete.

Recall the construction algorithm. In order to obtain $G_{k}$ with $k \geq 1$, we concatenate $G_{0}$ with $G_{k-1}^{(i)}$ for all $i \in\{1, \ldots, m\}$; thus, it is proper to regard $G_{k-1}^{(i)}$ as a subgraph of $G_{k}$. Set $V_{*}=V\left(G_{0}\right) \backslash\left\{v_{1}, \ldots, v_{m}\right\}$.

For $i=1, \ldots, m$, we can decompose the sum $\sum_{x \in G_{k-1}^{(i)}} \sum_{y \in G_{k}} d^{(k)}(x, y)$ into three terms:

$$
\begin{aligned}
& \sum_{x, y \in G_{k-1}^{(i)}} d^{(k)}(x, y)+\sum_{\substack{j=1 \\
j \neq i}}^{m} \sum_{x \in G_{k-1}^{(i)}, y \in G_{k-1}^{(j)}} d^{(k)}(x, y) \\
& +\sum_{x \in G_{k-1}^{(i)}, y \in V_{*}} d^{(k)}(x, y),
\end{aligned}
$$

and use the scaling mechanism for the edges and with the construction of $G_{k}$

$$
\begin{aligned}
= & f_{i} \Lambda_{k-1}+\left(\left(F+(m-2) f_{i}\right) N_{k-1} \lambda_{k-1}+N_{k-1}^{2} \alpha_{i}\right) \\
& +\left((n-m) f_{i} \lambda_{k-1}+N_{k-1} \beta_{i}\right),
\end{aligned}
$$

where

$$
\begin{aligned}
& \alpha_{i}=\sum_{j=1}^{m} d\left(v_{i}, v_{j}\right), \\
& \beta_{i}=\sum_{y \in V_{*}} d\left(v_{i}, y\right) .
\end{aligned}
$$

Similarly, we decompose the sum $\sum_{x \in V_{*}} \sum_{y \in G_{k}} d^{(k)}(x, y)$ into two terms:

$$
\sum_{x, y \in V_{*}} d^{(k)}(x, y)+\sum_{j=1}^{m} \sum_{x \in V_{*}} \sum_{y \in G_{k-1}^{(j)}} d^{(k)}(x, y),
$$

and use the scaling mechanism for the edges

$$
=\sum_{x, y \in V_{*}} d(x, y)+\left(N_{k-1} \sum_{x \in V_{*}} \sum_{j=1}^{m} d\left(x, v_{j}\right)+(n-m) F \lambda_{k-1}\right) \text {. }
$$

In conclusion, we obtain

$$
\begin{aligned}
\Lambda_{k}= & \sum_{x, y \in G_{k}} d^{(k)}(x, y)=\sum_{x \in V_{*}} \sum_{y \in G_{k}} d^{(k)}(x, y) \\
& +\sum_{i=1}^{m} \sum_{x \in G_{k-1}^{(i)}} \sum_{y \in G_{k}} d^{(k)}(x, y) \\
= & F \Lambda_{k-1}+2\left((m-1) N_{k-1}+n-m\right) F \lambda_{k-1}+N_{k-1}^{2} \alpha \\
& +2 N_{k-1} \beta+\eta,
\end{aligned}
$$

where

$$
\begin{aligned}
& \alpha=\sum_{i, j=1}^{m} d\left(v_{i}, v_{j}\right), \\
& \beta=\sum_{i=1}^{m} \sum_{y \in V_{*}} d\left(v_{i}, y\right), \\
& \eta=\sum_{x, y \in V_{*}} d(x, y) .
\end{aligned}
$$

Now, we define

$$
\Gamma_{k}=2\left((m-1) N_{k}+n-m\right) F \lambda_{k}+N_{k}^{2} \alpha+2 N_{k} \beta+\eta,
$$

for $k \geq 0$. Hence, we obtain the following key equation: 


$$
\Lambda_{k}=F \Lambda_{k-1}+\Gamma_{k-1}
$$

After all the preparations are complete, we are finally ready to study the asymptotic behavior of the average weighted geodesic distance of $G_{k}$. The result is stated in the following theorem.

Theorem 1. The asymptotic behavior of the average weighted geodesic distance of $G_{k}$ satisfies

$$
\begin{aligned}
\lim _{k \rightarrow \infty} \delta_{k}= & \frac{2(m-1) F}{\left(m^{2}-F\right)(m-F)} \sum_{i=1}^{m} d\left(v_{i}, s_{0}\right) \\
& +\frac{1}{m^{2}-F} \sum_{i, j=1}^{m} d\left(v_{i}, v_{j}\right) .
\end{aligned}
$$

Proof. First, we prove

$$
\lim _{k \rightarrow \infty} \delta_{k}=\frac{(m-1)^{2}}{m^{2}(n-1)^{2}\left(m^{2}-F\right)} \lim _{k \longrightarrow \infty} \frac{\Gamma_{k}}{m^{2 k}} .
$$

By equation (22), for all $k \geq 1$, we have

$$
\Lambda_{k}=\left(\sum_{t=0}^{k-1} F^{k-1-t} \Gamma_{t}\right)+F^{k} \Lambda_{0}
$$

Then,

$$
\begin{aligned}
\lim _{k \rightarrow \infty} \delta_{k} & =\lim _{k \rightarrow \infty} \frac{\Lambda_{k}}{m^{2 k}} \cdot \frac{m^{2 k}}{N_{k}\left(N_{k}-1\right)} \\
& =\frac{(m-1)^{2}}{m^{2}(n-1)^{2}} \lim _{k \longrightarrow \infty} \frac{\sum_{t=0}^{k-1} F^{k-1-t} \Gamma_{t}}{m^{2 k}} \\
& =\frac{(m-1)^{2}}{m^{2}(n-1)^{2} F} \lim _{k \longrightarrow \infty} \frac{\sum_{t=0}^{k-1} \Gamma_{t} / F^{t}}{\left(m^{2} / F\right)^{k}} \\
& =\frac{(m-1)^{2}}{m^{2}(n-1)^{2} F} \lim _{k \longrightarrow \infty} \frac{\Gamma_{k} / F^{k}}{\left(m^{2} / F\right)^{k+1}-\left(m^{2} / F\right)^{k}} \\
& =\frac{(m-1)^{2}}{m^{2}(n-1)^{2}\left(m^{2}-F\right)} \lim _{k \longrightarrow \infty} \frac{\Gamma_{k}}{m^{2 k}} .
\end{aligned}
$$

The last but one equality uses O. Stolz's formula. So, equation (24) is valid.

By the definition of $\Gamma_{k}$, one can verify that

$\lim _{k \rightarrow \infty} \frac{\Gamma_{k}}{m^{2 k}}=2(n-1) m F \lim _{k \rightarrow \infty} \frac{\lambda_{k}}{m^{k}}+\frac{m^{2}(n-1)^{2}}{(m-1)^{2}} \alpha$.

By equation (9) in Lemma 1, we have

$$
\lim _{k \longrightarrow \infty} \frac{\Gamma_{k}}{m^{2 k}}=\frac{2 m^{2}(n-1)^{2} F \lambda_{0}^{\prime}}{(m-F)(m-1)}+\frac{m^{2}(n-1)^{2}}{(m-1)^{2}} \alpha .
$$
result.
In networks $G_{k}$, let $p^{(k)}(x, y)$ denote the geodesic distance between nodes $x$ and $y$ in $G_{k}$. Set $p(\cdot, \cdot)=p^{(0)}(\cdot, \cdot)$ for convention. Define

$$
\Theta_{k}=\sum_{x, y \in G_{k}} p^{(k)}(x, y)
$$

for $k \geq 0$. Thus, the average geodesic distance of $G_{k}$ is given by

$$
\theta_{k}=\frac{\Theta_{k}}{N_{k}\left(N_{k}-1\right)}
$$

We can also compute $\theta_{k}$, formally obtained by setting $f_{1}=\cdots=f_{m}=1$ and replacing weighted geodesic distance $d^{(k)}(\cdot, \cdot)$ by geodesic distance $p^{(k)}(\cdot, \cdot)$ in the previous argument. The asymptotic behavior of the average geodesic distance of $G_{k}$ is given in the following theorem, and the theorem shows the average geodesic distance increases logarithmically with the network size.

Theorem 2. The asymptotic behavior of the average geodesic distance of $G_{k}$ satisfies

$$
\begin{gathered}
\lim _{k \longrightarrow \infty}\left(\theta_{k}-\frac{2 k}{m} \sum_{i=1}^{m} p\left(v_{i}, s_{0}\right)\right)=\frac{\sum_{i, j=1}^{m} p\left(v_{i}, v_{j}\right)}{m(m-1)} \\
+\frac{2(m-1)}{m(n-1)}\left(\sum_{x \in G_{0}} p\left(x, s_{0}\right)+\frac{\sum_{x \in V_{*}} p\left(x, s_{0}\right)}{m-1}\right) \\
-2\left(\frac{n-m}{m(m-1)(n-1)}+\frac{1}{m-1}\right) \sum_{i=1}^{m} p\left(v_{i}, s_{0}\right) .
\end{gathered}
$$

Proof. Define

$$
\begin{aligned}
& \iota_{k}=\sum_{x \in G_{k}} p^{(k)}\left(x, s_{k}\right), \quad \text { for } k \geq 0, \\
& \iota_{0}^{\prime}=\sum_{i=1}^{m} p\left(v_{i}, s_{0}\right), \quad \iota_{0}^{\prime \prime}=\iota_{0}-\iota_{0}^{\prime} .
\end{aligned}
$$

By equation (8), we obtain

$$
\begin{aligned}
\iota_{k} & =m^{k} \iota_{0}+\left(\sum_{t=0}^{k-1} m^{k-1-t} N_{t}\right) \iota_{0}^{\prime}+\left(\sum_{t=0}^{k-1} m^{t}\right) \iota_{0}^{\prime \prime} \\
& =m^{k} \iota_{0}-\frac{m^{k}(n-m) \iota_{0}^{\prime}}{m(m-1)} \sum_{t=0}^{k-1} \frac{1}{m^{t}}+\left(\sum_{t=0}^{k-1} m^{t}\right) \iota_{0}^{\prime \prime}+\frac{m^{k}(n-1) \iota_{0}^{\prime}}{m-1} k \\
& =\tau_{k}+\frac{m^{k}(n-1) \iota_{0}^{\prime}}{m-1} k,
\end{aligned}
$$

for $k>0$. Take $\iota_{0}=\tilde{\iota}_{0}$. One can verify that

$$
\lim _{k \rightarrow \infty} \frac{\tilde{\iota}_{k}}{m^{k}}=\iota_{0}-\frac{n-m}{(m-1)^{2}} l_{0}^{\prime}+\frac{1}{m-1} \iota_{0}^{\prime \prime} .
$$

Now, we define 


$$
\begin{aligned}
& \Upsilon_{k}=2\left((m-1) N_{k}+n-m\right) m \iota_{k}+N_{k}^{2} \alpha_{*}+2 N_{k} \beta_{*}+\eta_{*}, \\
& \widetilde{\Upsilon}_{k}=2\left((m-1) N_{k}+n-m\right) \tilde{m \tau}_{k}+N_{k}^{2} \alpha_{*}+2 N_{k} \beta_{*}+\eta_{*},
\end{aligned}
$$

for $k \geq 0$. The definitions of $\alpha_{*}, \beta_{*}$, and $\eta_{*}$ are similar to $\alpha, \beta$, and $\eta$, respectively; we just replace $d(\cdot, \cdot)$ by geodesic distance $p(\cdot, \cdot)$ in the new definitions. Thus,

$$
\Upsilon_{k}=\widetilde{\Upsilon}_{k}+U_{k}\left(\iota_{k}-\tilde{\iota}_{k}\right)=\widetilde{\Upsilon}_{k}+U_{k} \frac{m^{k}(n-1) \iota_{0}^{\prime}}{m-1} k,
$$

where $U_{k}=2\left((m-1) N_{k}+n-m\right) m$ for $k \geq 0$.

By equations (22) and (25), we obtain

$$
\Theta_{k}=\left(\sum_{t=0}^{k-1} m^{k-1-t} \Upsilon_{t}\right)+m^{k} \Theta_{0} .
$$

Since $\lim _{k \longrightarrow \infty}\left(m^{k} \Theta_{0} / N_{k}\left(N_{k}-1\right)\right)=0$, we have

$$
\begin{aligned}
& \lim _{k \rightarrow \infty}\left(\theta_{k}-\frac{2 \iota_{0}^{\prime}}{m}(k-1)+\frac{2 \iota_{0}^{\prime}}{m(m-1)}\right) \\
& \quad=\lim _{k \rightarrow \infty}\left(\frac{\left(\sum_{t=0}^{k-1} m^{k-1-t} \Upsilon_{t}\right)}{N_{k}\left(N_{k}-1\right)}-\frac{2 \iota_{0}^{\prime}}{m}(k-1)+\frac{2 \iota_{0}^{\prime}}{m(m-1)}\right) \\
& =\lim _{k \rightarrow \infty}\left(\frac{\left(\sum_{t=0}^{k-1} m^{k-1-t}\left(\widetilde{\Upsilon}_{t}+U_{t}\left(\iota_{t}-\widetilde{\iota}_{t}\right)\right)\right)}{N_{k}\left(N_{k}-1\right)}-\frac{2 \iota_{0}^{\prime}}{m}(k-1)+\frac{2 \iota_{0}^{\prime}}{m(m-1)}\right) \\
& \quad=\lim _{k \rightarrow \infty} \frac{\sum_{t=0}^{k-1} m^{k-1-t} \widetilde{\Upsilon}_{t}}{N_{k}\left(N_{k}-1\right)}+\lim _{k \rightarrow \infty}\left(\frac{\sum_{t=0}^{k-1} m^{k-1-t} U_{t}\left(\iota_{t}-\widetilde{\imath}_{t}\right)}{N_{k}\left(N_{k}-1\right)}-\frac{2 \iota_{0}^{\prime}}{m}(k-1)+\frac{2 \iota_{0}^{\prime}}{m(m-1)}\right) .
\end{aligned}
$$

By direct calculation, we have

$\lim _{k \longrightarrow \infty}\left(\frac{\sum_{t=0}^{k-1} m^{k-1-t} U_{t}\left(\iota_{t}-\widetilde{\iota}_{t}\right)}{N_{k}\left(N_{k}-1\right)}-\frac{2 \iota_{0}^{\prime}}{m}(k-1)+\frac{2 \iota_{0}^{\prime}}{m(m-1)}\right)=0$.

Thus,

$$
\begin{aligned}
& \lim _{k \rightarrow \infty}\left(\theta_{k}-\frac{2 \iota_{0}^{\prime}}{m}(k-1)+\frac{2 \iota_{0}^{\prime}}{m(m-1)}\right) \\
& =\lim _{k \rightarrow \infty} \frac{\sum_{t=0}^{k-1} m^{k-1-t} \widetilde{\Upsilon}_{t}}{N_{k}\left(N_{k}-1\right)} \\
& =\frac{(m-1)^{2}}{m^{3}(n-1)^{2}} \lim _{k \longrightarrow \infty} \frac{\sum_{t=0}^{k-1} \widetilde{\Upsilon}_{t} / m^{t}}{m^{k}} \\
& =\frac{m-1}{m^{3}(n-1)^{2}} \lim _{k \rightarrow \infty} \frac{\widetilde{\Upsilon}_{k}}{m^{2 k}} .
\end{aligned}
$$

By equation (34), we obtain

$$
\begin{aligned}
\lim _{k \rightarrow \infty} \frac{\widetilde{Y}_{k}}{m^{2 k}=} & 2 m^{2}(n-1)\left(l_{0}-\frac{n-m}{(m-1)^{2}} l_{0}^{\prime}+\frac{1}{m-1} l_{0}^{\prime \prime}\right) \\
& +\frac{m^{2}(n-1)^{2}}{(m-1)^{2}} \alpha_{*} .
\end{aligned}
$$

Combining equations (40) and (41), we have the desired result. The proof is complete.
3.2. Node Strength Distribution. Given node $x$ in $G_{k}$, we denote by $N^{(k)}(x)$ the set of nodes adjacent to $x$. If $y \in N^{(k)}(x)$, the weight of edge $(x y)$ in $G_{k}$ is $w_{x y}^{(k)}$. The node strength of $x$ is defined by

$$
w_{x}^{(k)}=\sum_{y \in N^{(k)}(x)} w_{x y}^{(k)} .
$$

Node strength is a generalization of the degree to weighted graphs. We are in the place to study the node strength distribution of the weighted graphs $G_{k}$. Let $g_{k}(h)$ denote the cardinality of nodes in $G_{k}$ that have strength $h$. Without loss of generality, we assume that $n-m-1=l>0$ and $V\left(G_{0}\right) \backslash\left\{s_{0}, v_{1}, \ldots, v_{m}\right\}=\left\{u_{1}, \ldots, u_{l}\right\}$.

We first present some notations and definitions that will be used throughout this section. Set

$$
\begin{aligned}
& x_{0}=w_{s_{0}}^{(0)}, z_{j}=w_{u_{j}}^{(0)}, \quad \text { for } j=1, \ldots, l, \\
& y_{i}=w_{v_{i}}^{(0)}, x_{i}=y_{i}+f_{i} x_{0}, \quad \text { for } i=1, \ldots, m .
\end{aligned}
$$

Recall that $\mathbb{N}=\{0,1,2, \ldots\}$. Define

$$
\begin{aligned}
\Pi_{k} & =\left\{\left(r_{1}, \ldots, r_{m}\right) \in \mathbb{N}^{m}: r_{1}+\cdots+r_{m}=k\right\}, \\
\Pi & =\bigcup_{k=0}^{\infty} \Pi_{k} .
\end{aligned}
$$

For $\Delta \subset \Pi$, we define $\Delta_{k}=\left\{\left(r_{1}, \ldots, r_{m}\right) \in \Delta: r_{1}+\cdots+r_{m}=k\right\}, \quad$ for $k \geq 0$.

For $\Delta \subset \Pi_{k}$, we can also define 


$$
\begin{aligned}
\Delta^{t} & =\left\{\left(r_{1}, \ldots, r_{m}\right) \in \Pi_{t}: \text { there exists }\left(r_{1}^{\prime}, \ldots, r_{m}^{\prime}\right)\right. \\
& \left.\in \Delta \text { s.t. }\left(r_{1}, \ldots, r_{m}\right) \prec\left(r_{1}^{\prime}, \ldots, r_{m}^{\prime}\right)\right\},
\end{aligned}
$$

for $0 \leq t \leq k$. Here, $\left(r_{1}, \ldots, r_{m}\right)<\left(r_{1}^{\prime}, \ldots, r_{m}^{\prime}\right)$ means $r_{i} \leq r_{i}^{\prime}$ for all $i \in\{1, \ldots, m\}$.

Lemma 3. If $\Delta \subset \Pi_{k}$ for $k>0$, then for any $0 \leq t<k$, we have

$$
\begin{aligned}
& \sum_{\left(r_{1}, \ldots, r_{m}\right) \in \Delta^{t}} \frac{t !}{r_{1} ! \cdots r_{m} !}\left(f_{1}^{r_{1}} \cdots f_{m}^{r_{m}}\right)^{d_{\text {fract }}} \\
\geq & \sum_{\left(r_{1}, \ldots, r_{m}\right) \in \Delta} \frac{k !}{r_{1} ! \cdots r_{m} !}\left(f_{1}^{r_{1}} \cdots f_{m}^{r_{m}}\right)^{d_{\text {fract }}} .
\end{aligned}
$$

Proof. For any $\Omega \subset \Pi$, we define

$$
S(\Omega)=\sum_{\left(r_{1}, \ldots, r_{m}\right) \in \Omega} \frac{\left(r_{1}+\cdots+r_{m}\right) !}{r_{1} ! \cdots r_{m} !}\left(f_{1}^{r_{1}} \cdots f_{m}^{r_{m}}\right)^{d_{\text {fract }}},
$$

for brevity. Note that

$$
\begin{aligned}
1= & \left(f_{1}^{d_{\text {fract }}}+\cdots+f_{m}^{d_{\text {fract }}}\right)^{k} \\
= & S\left(\Delta^{t}\right)\left(f_{1}^{d_{\text {fract }}}+\cdots+f_{m}^{d_{\text {fract }}}\right)^{k-t} \\
& +S\left(\Pi_{t} \backslash \Delta^{t}\right)\left(f_{1}^{d_{\text {fract }}}+\cdots+f_{m}^{d_{\text {fract }}}\right)^{k-t} \\
= & S(\Delta)+S\left(\Pi_{k} \backslash \Delta\right) .
\end{aligned}
$$

By the definition of $\Delta^{t}$, we have the desired result.

Lemma 4. For any $\left(\alpha_{1}, \ldots, \alpha_{m}\right) \in \Pi$, we define

$$
\Delta_{\left(\alpha_{1}, \ldots, \alpha_{m}\right)}=\left\{\left(r_{1}, \ldots, r_{m}\right) \in \Pi: f_{1}^{r_{1}} \cdots f_{m}^{r_{m}}=f_{1}^{\alpha_{1}} \cdots f_{m}^{\alpha_{m}}\right\} .
$$

Then, we have

$$
S\left(\Delta_{\left(\alpha_{1}, \ldots, \alpha_{m}\right)}\right) \leq 1
$$

Proof. For fixed $\left(\alpha_{1}, \ldots, \alpha_{m}\right)$, we let $\Delta=\Delta_{\left(\alpha_{1}, \ldots, \alpha_{m}\right)}$ for brevity. There exist integers $t_{0}, \ldots, t_{l}$ such that

$$
\begin{aligned}
& 0 \leq t_{0}<t_{1}<\cdots<t_{l}, \\
& \Delta=\bigcup_{i=0}^{l} \Delta_{t_{i}} .
\end{aligned}
$$

Define a sequence of sets $\Omega_{0}, \ldots, \Omega_{l}$ satisfying $\Omega_{l}=\Delta_{t_{l}}$ and

$$
\Omega_{i}=\left(\Omega_{i+1}\right)^{t_{i}} \cup \Delta_{t_{i}}, \text { for } i=0, \ldots, l-1 .
$$

Firstly, we prove

$$
\left(\Omega_{i+1}\right)^{t_{i}} \cap \Delta_{t_{i}}=\varnothing,
$$

for $i=0, \ldots, l-1$. If $\left(r_{1}, \ldots, r_{m}\right) \in \Omega_{i+1}$, then $f_{1}^{r_{1}} \cdots f_{m}^{r_{m}} \geq f_{1}^{\alpha_{1}} \cdots f_{m}^{\alpha_{m}}$. Since $\quad t_{i}<t_{i+1}$, any $\left(r_{1}, \ldots, r_{m}\right) \in\left(\Omega_{i+1}\right)^{t_{i}}$ satisfies that $f_{1}^{r_{1}} \cdots f_{m}^{r_{m}}>f_{1}^{\alpha_{1}} \cdots f_{m}^{\alpha_{m}}$. Hence, $\left(\Omega_{i+1}\right)^{t_{i}}$ and $\Delta_{t_{i}}$ have no common member.
By Lemma 3, we obtain

$$
\begin{aligned}
S(\Delta) & =\sum_{i=0}^{l} S\left(\Delta_{t_{i}}\right) \\
& \leq \sum_{i=0}^{l-2} S\left(\Delta_{t_{i}}\right)+S\left(\Delta_{t_{l-1}}\right)+S\left(\left(\Delta_{t_{l}}\right)^{t_{l-1}}\right) \\
& =\sum_{i=0}^{l-2} S\left(\Delta_{t_{i}}\right)+S\left(\Omega_{l-1}\right) \\
& \ldots \ldots \\
& \leq S\left(\Omega_{0}\right) \leq 1 .
\end{aligned}
$$

The proof is complete.

Theorem 3. There exists $C_{1}>0$ such that, for all $k>0$, we have

$$
g_{k}(h) \leq \frac{C_{1}}{h^{d_{\text {fract }}}} .
$$

If $f_{1}=\cdots=f_{m}=f$, then there exists $C_{2}>0$ such that, for all $k>0$, if $g_{k}(h) \neq 0$, then

$$
g_{k}(h) \geq \frac{C_{2}}{h^{d_{\text {fract }}}} .
$$

Proof. For any $h>0$, we set

$$
\begin{aligned}
& \phi_{j}=\left\{\left(r_{1}, \ldots, r_{m}\right) \in \bigcup_{t=0}^{k} \Pi_{t}: h=z_{j} f_{1}^{r_{1}} \cdots f_{m}^{r_{m}}\right\}, \quad \text { for } j=1, \ldots, l, \\
& \varphi_{i}=\left\{\left(r_{1}, \ldots, r_{m}\right) \in \bigcup_{t=0}^{k-1} \Pi_{t}: h=x_{i} f_{1}^{r_{1}} \cdots f_{m}^{r_{m}}\right\}, \quad \text { for } i=1, \ldots, m, \\
& \eta_{i}=\left\{\left(r_{1}, \ldots, r_{m}\right) \in \Pi_{k}: h=y_{i} f_{1}^{r_{1}} \cdots f_{m}^{r_{m}}\right\}, \quad \text { for } i=1, \ldots, m .
\end{aligned}
$$

One can easily verify that

$$
g_{k}(h) \leq \frac{x_{0}^{d_{\text {fract }}}}{h^{d_{\text {fract }}}}+\sum_{j=1}^{l} \frac{S\left(\phi_{j}\right)}{\left(h / z_{j}\right)^{d_{\text {fract }}}}+\sum_{i=1}^{m} \frac{S\left(\varphi_{i}\right)}{\left(h / x_{i}\right)^{d_{\text {fract }}}}+\sum_{i=1}^{m} \frac{S\left(\eta_{i}\right)}{\left(h / y_{i}\right)^{d_{\text {fract }}}} \text {. }
$$

By equation (51), we have

$$
g_{k}(h) \leq \frac{\sum_{i=0}^{m} x_{i}^{d_{\text {fract }}}+\sum_{i=1}^{m} y_{i}^{d_{\text {fract }}}+\sum_{j=1}^{l} z_{j}^{d_{\text {fract }}}}{h^{d_{\text {fract }}}}=: \frac{C_{1}}{h^{d_{\text {fract }}}} \text {. }
$$

Now, we assume $f_{1}=\cdots=f_{m}=f$. There are at least $m^{t}$ nodes that have strength $f^{t} z_{j}$ for $0 \leq t \leq k$. There are at least $m^{t}$ nodes that have strength $f^{t} x_{i}$ for $0 \leq t<k$. There are at least $m^{k}$ nodes that have strength $f^{k} y_{i}$. Set

$$
C_{2}=\left(\min \left\{x_{0}, y_{i}, z_{j}: i=1, \ldots, m, j=1, \ldots, l\right\}\right)^{d_{\text {fract }}} .
$$

If $h=z_{j} f^{t}$, then

$$
g_{k}(h) \geq m^{t}=\frac{1}{\left(h / z_{j}\right)^{d_{\text {fract }}}} \geq \frac{C_{2}}{h^{d_{\text {fract }}}} .
$$

If $h=y_{i} f^{k}$, then 


$$
g_{k}(h) \geq m^{k}=\frac{1}{\left(h / y_{i}\right)^{d_{\text {fract }}}} \geq \frac{C_{2}}{h^{d_{\text {fract }}}} .
$$

If $h=x_{i} f^{t}$, for $i>0$, then

$$
g_{k}(h) \geq m^{t}=\frac{1}{\left(h / x_{i}\right)^{d_{\text {fract }}}}=\frac{\left(y_{i}+f x_{0}\right)^{d_{\text {fract }}}}{h^{d_{\text {fract }}}} \geq \frac{C_{2}}{h^{d_{\text {fract }}}} .
$$

The proof is complete.

\section{Data Availability}

The data used to support the findings of this study are available from the corresponding author upon request.

\section{Conflicts of Interest}

The authors declare that they have no conflicts of interest.

\section{Acknowledgments}

This work was supported by the Scientific Research Fund of the Education Department of Hunan Province (Grant no. 19K019) and the Hunan Provincial Natural Science Fund (Grant no. 2020JJ4163).

\section{References}

[1] S. Boccaletti, V. Latora, Y. Moreno, M. Chavez, and D. Hwang, "Complex networks: structure and dynamics," Physics Reports, vol. 424, no. 4-5, pp. 175-308, 2006.

[2] S. Dorogovtsev, Lectures On Complex Networks, Oxford University Press, Oxford, UK, 2010.

[3] R. Huang, "A qd-type method for computing generalized singular values of BF matrix pairs with sign regularity to high relative accuracy," Mathematics of Computation, vol. 89, no. 321, pp. 229-252, 2020.

[4] M. E. J. Newman, "The structure and function of complex networks," SIAM Review, vol. 45, no. 2, pp. 167-256, 2003.

[5] A. Barrat, M. Barthelemy, R. Pastor-Satorras, and A. Vespignani, "The architecture of complex weighted networks," Proceedings of the National Academy of Sciences, vol. 101, no. 11, pp. 3747-3752, 2004.

[6] A.-L. Barabási, E. Ravasz, and T. Vicsek, "Deterministic scalefree networks," Physica A: Statistical Mechanics and Its Applications, vol. 299, no. 3-4, pp. 559-564, 2001.

[7] Z.-Z. Zhang, S.-G. Zhou, T. Zou, L. Chen, and J. Guan, "Selfsimilarity, small-world, scale-free scaling, disassortativity, and robustness in hierarchical lattices," The European Physical Journal B, vol. 56, no. 3, pp. 259-271, 2007.

[8] Z. Zhang, S. Gao, L. Chen, S. Zhou, H. Zhang, and J. Guan, "Mapping koch curves into scale-free small-world networks," Journal of Physics A: Mathematical and Theoretical, vol. 43, no. 39, Article ID 395101, 2010.

[9] T. Carletti and S. Righi, "Weighted fractal networks," Physica A: Statistical Mechanics and Its Applications, vol. 389, no. 10, pp. 2134-2142, 2010.

[10] J. Hutchinson, "Fractals and self-similarity," Indiana University Mathematics Journal, vol. 30, no. 5, pp. 713-747, 1981.

[11] T. Li, K. Jiang, and L. Xi, "Average distance of self-similar fractal trees,” Fractals, vol. 26, no. 1, Article ID 1850016, 2018.
[12] L. Xi and Q. Ye, "Average distances on substitution trees," Physica A: Statistical Mechanics and Its Applications, vol. 529, Article ID 121556, 2019.

[13] Y. Xiao and J. Gu, "Uniform Cantor sets as hyperbolic boundaries," Filomat, vol. 28, no. 8, pp. 1737-1745, 2014.

[14] J. E. Schneider, "A generalization of the von Koch curve," Mathematics Magazine, vol. 38, no. 3, pp. 144-147, 1965.

[15] K. Falconer, Fractal Geometry, John Wiley \& Sons, Chichester, UK, 1990.

[16] A. Bondy and M. Ram Murty, Graph Theory, Springer-Verlag London, London, UK, 2008. 